\title{
Stabilization of Planar Collective Motion: All-to-All Communication
}

\author{
Rodolphe Sepulchre, Member, IEEE, Derek A. Paley, Student Member, IEEE, and \\ Naomi Ehrich Leonard, Fellow, IEEE
}

\begin{abstract}
This paper proposes a design methodology to stabilize isolated relative equilibria in a model of all-to-all coupled identical particles moving in the plane at unit speed. Isolated relative equilibria correspond to either parallel motion of all particles with fixed relative spacing or to circular motion of all particles with fixed relative phases. The stabilizing feedbacks derive from Lyapunov functions that prove exponential stability and suggest almost global convergence properties. The results of the paper provide a low-order parametric family of stabilizable collectives that offer a set of primitives for the design of higher-level tasks at the group level.
\end{abstract}

Index Terms-Cooperative control, geometric control, multiagent systems, stabilization.

\section{INTRODUCTION}

$\mathbf{T}$ HE collective control of multiagent systems is a rapidly developing field, motivated by a number of engineering applications that require the coordination of a group of individually controlled systems. Applications include formation control of unmanned aerial vehicles (UAVs) [1], [2] and spacecraft [3], cooperative robotics [4]-[6], and sensor networks [7], [8]. A specific application motivating the results of the present paper is the use of autonomous underwater vehicles (AUVs) to collect oceanographic measurements in formations or patterns that maximize the information intake, see e.g., [9], [10]. This can be achieved by matching the measurement density in space and time to the characteristic scales of the oceanographic process of interest. Coordinated, periodic trajectories such as the ones studied in this paper, provide a means to collect measurements with the desired spatial and temporal separation.

The design focus of collective stabilization problems is on achieving a certain level of synchrony among possibly many but individually controlled dynamical systems. The primary design

Manuscript received June 27, 2005; revised February 20, 2006 and July 24, 2006. Recommended by Associate Editor F. Bullo. The work of D. A. Paley was supported by the National Science Foundation Graduate Research Fellowship, the Princeton University Gordon Wu Graduate Fellowship, and the Pew Charitable Trust under Grant 2000-002558. The work of N. E. Leonard was supported in part by the Office of Naval Research under Grants N00014-02-1-0861 and N00014-04-1-0534. This paper presents research results of the Belgian Network DYSCO (Dynamical Systems, Control, and Optimization), funded by the Interuniversity Attraction Poles Programme, initiated by the Belgian State, Science Policy Office. The scientific responsibility rests with its author(s).

R. Sepulchre is with the Departement of Electrical Engineering and Computer Science, Université de Liège, 4000 Liège Sart-Tilman, Belgium (e-mail: r.sepulchre@ulg.ac.be).

D. A. Paley and N. E. Leonard are with the Department of Mechanical and Aerospace Engineering, Princeton University, Princeton, NJ 08544 USA (e-mail: dpaley@ princeton.edu; naomi@ princeton.edu).

Digital Object Identifier 10.1109/TAC.2007.898077 issue is not how to control the individual dynamics, but rather how to interconnect them to achieve the desired level of synchrony. This motivates the use of simplified models for the individuals. For instance, synchrony in populations of coupled oscillators has been studied primarily by means of phase models; these models only retain the phase information of individual oscillators as the fundamental information pertaining to synchrony measures of the ensemble [11]. Even these simplified models raise challenging issues for the analysis or design of their interconnection. They have recently motivated a number of new developments in stability analysis [12], [13]. The interconnected control system is high dimensional, both in the number of state variables and control variables. It is nevertheless characterized by a high level of symmetry, which is maximal when all the individual models are assumed identical. Symmetry properties make these models well suited to the reduction techniques of geometric control [14]. Symmetry and geometry play a central role in the analysis of cyclic pursuit strategies for kinematic unicycle models studied in [15].

Motivated by the issues above, we consider in the present paper the model of identical, all-to-all coupled, planar particles introduced in [16]. The particles move at constant speed and are subject to steering controls that change their orientation. In addition to a phase variable that models the orientation of the velocity vector, the state of each particle includes its position in the plane. The synchrony of the collective motion is thus measured both by the relative phasing and the relative spacing of particles.

In [17] and [18], we observed that the norm of the average linear momentum of the group is a key control parameter: it is maximal in the case of parallel motions of the group and minimal in the case of circular motions around a fixed point. We exploited the analogy with phase models of coupled oscillators to design steering control laws that stabilize either parallel or circular motions. Expanding on this idea, the design methodology in the present paper is to construct potentials that reach their minimum at desired collective formations and to derive the corresponding gradient-like steering control laws as stabilizing feedbacks. We treat separately phase potentials that control the relative orientation of particles and spacing potentials that control their relative spacing. We show that the design can be made somewhat systematic and versatile, resulting in a simple but general controller structure. The stabilizing feedbacks depend on a restricted number of parameters that control the shape and the level of symmetry of parallel or circular formations. This low-order parametric family of stabilizable collectives offers a set of primitives that can be used to solve path planning or optimization tasks at the group level. 
The results of the paper rest on two idealistic assumptions: all-to-all communication and identical individuals. Neither of these assumptions is realistic in a practical environment, not in engineering models nor in models of natural groups. The all-to-all assumption is instrumental to the results of the paper but this strong assumption is completely relaxed in a companion paper [30] where we extend the present results to communication topologies where communication is limited. The assumption of identical individuals is fundamental to the symmetry properties of the closed-loop vector field, which is instrumental to the proposed stability analysis. It is known that the individual dynamics may exhibit much more complicated behaviors away from this ideal situation. Many earlier studies nevertheless suggest that synchrony is robust and that an ensemble phenomenon resulting from a specific interconnection structure will persist in spite of individual discrepancies. The analysis of the celebrated Kuramoto model (see [19] for a recent review) exemplifies both the robustness of synchronization at the ensemble level and its mathematical mysteries at the individual level in a population of non-identical, all-to-all coupled oscillators. In this sense, the ideally engineered models considered in this paper may help in capturing gross dynamical properties of more realistic, multiagent, simulation models or of biologists' observations of animal groups.

The rest of this paper is organized as follows. Section II reviews the geometric properties of the considered model. Section III introduces a basic phasing potential that controls the group linear momentum. In Section IV we introduce a spacing potential that is minimum in circular formations. These results provide the basic control laws to achieve either parallel or circular formations, the only possible relative equilibria of the model. Due to symmetry, the dimension of the equilibrium set is high and can be reduced with the help of symmetry breaking controls laws that derive from further potentials. We show in Sections V, VI and VII how to stabilize isolated relative equilibria of the model, both in circular formations (Sections V and VI) and in parallel formations (Section VII). Exponential stabilization of isolated circular relative equilibria is presented in Section VI. Section VIII illustrates how to combine the results of the previous sections in a low-parameter catalog of stabilizable collectives. Conclusions are presented in Section IX.

\section{A Model of SteEred Particles In the Plane}

We consider a continuous-time kinematic model of $N$ identical particles (of unit mass) moving in the plane at unit speed [1]

$$
\begin{aligned}
& \dot{r}_{k}=e^{i \theta_{k}} \\
& \dot{\theta}_{k}=u_{k}, \quad k=1, \ldots, N .
\end{aligned}
$$

In complex notation, the vector $r_{k}=x_{k}+i y_{k} \in \mathbb{C} \approx \mathbb{R}^{2}$ denotes the position of particle $k$ and the angle $\theta_{k} \in S^{1}$ denotes the orientation of its (unit) velocity vector $e^{i \theta_{k}}=\cos \theta_{k}+$ $i \sin \theta_{k}$. The scalar $u_{k}$ is the steering control for particle $k$. We use a bold variable without index to denote the corresponding $N$-vector, e.g., $\boldsymbol{\theta}=\left(\theta_{1}, \ldots, \theta_{N}\right)^{T}$ and $\mathbf{u}=\left(u_{1}, \ldots, u_{N}\right)^{T}$. In the absence of steering control $\left(\dot{\theta}_{k}=0\right)$, each particle moves at unit speed in a fixed direction and its motion is decoupled from the other particles. We study the design of various feedback control laws that result in coupled dynamics and closed-loop convergence to different types of organized or collective motion. We assume identical control for each particle. In that sense, the collective motions that we analyze in the present paper do not require differentiated control action for the different particles (e.g., the presence of a leader for the group).

The model (1) has been recently studied by Justh and Krishnaprasad [1]. These authors have emphasized the Lie group structure that underlies the state space. The configuration space consists of $N$ copies of the group $\mathrm{SE}(2)$. When the control law only depends on relative orientations and relative spacing, i.e., on the variables $\theta_{k j}=\theta_{k}-\theta_{j}$ and $r_{k j}=r_{k}-r_{j}, j, k=1, \ldots, N$, the closed-loop vector field is invariant under an action of the symmetry group $\mathrm{SE}(2)$ and the closed-loop dynamics evolve on a reduced quotient manifold. This $(3 N-3)$-dimensional manifold is called the shape space and it corresponds to the space of all relative orientations and relative positions. Equilibria of the reduced dynamics are called relative equilibria and can be only of two types [1]: parallel motions, characterized by a common orientation for all the particles (with arbitrary relative spacing), and circular motions, characterized by circular orbits of the particles around a fixed point. Both types of motion have been observed in simulations in a number of models that are kinematic or dynamic variants of the model (1), see for instance [20].

A simplification of the model (1) occurs when the feedback laws depend on relative orientations only. The control has then a much larger symmetry group ( $N$ copies of the translation group), and the reduced model becomes a pure phase model $\dot{\boldsymbol{\theta}}=\mathbf{u}$ where the phase variable $\boldsymbol{\theta}$ belongs to the $N$-dimensional torus $T^{N}$. This phase model still has an $S^{1}$-symmetry if the feedback only depends on phase differences. Phase-oscillator models of this type have been widely studied in the neuroscience and physics literature. They represent a simplification of more complex oscillator models in which the uncoupled oscillator dynamics each have an attracting limit cycle in a higher-dimensional state space. Under the assumption of weak coupling, the reduction of higher-dimensional models to phase models by asymptotic methods (singular perturbations and averaging) has been studied in e.g., Ermentrout and Kopell [21] and Hoppensteadt and Izhikevich [22].

The results in this paper build upon an extensive literature on phase models of coupled oscillators [11], [19], [23], [24]. We will stress the close connection between collective motions in groups of oscillators and collective motions in groups of moving particles.

Euler discretization of the continuous-time model (1) yields the discrete-time equations

$$
\begin{aligned}
& r_{k}(t+1)-r_{k}(t)=e^{i \theta_{k}(t)} \\
& \theta_{k}(t+1)-\theta_{k}(t)=u_{k}(t), \quad k=1, \ldots, N
\end{aligned}
$$

The direction of motion of particle $k$ is updated at each time step according to some feedback control $u_{k}$. We consider continuous-time models in this paper, but we mention a few relevant references that study their discrete-time counterpart. Couzin et al. [25] have studied such a model where the feedback 
control is determined from a set of simple rules: repulsion from close neighbors, attraction to distant neighbors, and preference for a common orientation. Their model includes stochastic effects but also exhibits collective motions reminiscent of either parallel motion or circular motion around a fixed center of mass. Interestingly, these authors have shown coexistence of these two types of motion in certain parameter ranges and hysteretic transition from one to the other. A discrete stochastic phase model has been studied by Vicsek et al. [26].

\section{Phase Stabilization}

We start our analysis with a basic but key observation for the results of this paper: the (average) linear momentum $\dot{R}$ of a group of particles satisfying (1) is the centroid of the phase particles $p_{\theta}$, that is

$$
\dot{R}=\frac{1}{N} \sum_{k=1}^{N} \dot{r}_{k}=\frac{1}{N} \sum_{k=1}^{N} e^{i \theta_{k}} \triangleq p_{\theta}=\left|p_{\theta}\right| e^{i \Psi} .
$$

The parameter $\left|p_{\theta}\right|$ is a measure of synchrony of the phase variables $\boldsymbol{\theta}$ [11], [19]. It is maximal when all phases are synchronized (identical). It is minimal when the phases balance to result in a vanishing centroid. The set of synchronized states is an isolated point modulo the action of the symmetry group $S^{1}$. It defines a manifold of dimension one. The set of balanced states, which we call the balanced set, is defined as all $\boldsymbol{\theta} \in T^{N}$ for which $p_{\theta}=0$. For $N$ odd, the equation $p_{\theta}=0$ has full rank everywhere and the set of balanced states defines a manifold of codimension two. For $N$ even, the balanced set is not a manifold of codimension two. The equation loses rank at points where there are two anti-synchronized, equally-sized clusters, i.e., each cluster consists of $(N / 2)$ synchronized phases and the phase of one cluster equals the phase of the other cluster plus $\pi$.

In the particle model (1), synchronization of the phases corresponds to a parallel formation: all particles move in the same direction. In contrast, balancing of the phases corresponds to collective motion around a fixed center of mass. Control of the group linear momentum is thus achieved by minimizing or maximizing the potential

$$
U_{1}(\boldsymbol{\theta})=\frac{N}{2}\left|p_{\theta}\right|^{2},
$$

which suggests the gradient control $\mathbf{u}=-K \operatorname{grad} U_{1}$, i.e.,

$$
u_{k}=-K \frac{\partial U_{1}}{\partial \theta_{k}}=-K\left\langle p_{\theta}, i e^{i \theta_{k}}\right\rangle=-\frac{K}{N} \sum_{j=1}^{N} \sin \theta_{j k} .
$$

The inner product $\langle\cdot, \cdot\rangle$ is defined by $\left\langle\mathrm{z}_{1}, \mathrm{z}_{2}\right\rangle=\operatorname{Re}\left\{\bar{z}_{1} z_{2}\right\}$ for $z_{1}, z_{2} \in \mathbb{C}$. For vectors, we use the analogous boldface notation $\left\langle\mathbf{z}_{1}, \mathbf{z}_{\mathbf{2}}\right\rangle=\operatorname{Re}\left\{\overline{\mathbf{z}}_{1}^{\mathrm{T}} \mathbf{z}_{2}\right\}$ for $\mathbf{z}_{1}, \mathbf{z}_{\mathbf{2}} \in \mathbb{C}^{N}$. This all-to-all sinusoidal coupling (5) is the most frequently studied coupling in the literature of coupled oscillators [11], [19], [24]. Its gradient nature enables the following global convergence analysis.

Theorem 1: The potential $U_{1}=(N / 2)\left|p_{\theta}\right|^{2}$ reaches its unique minimum when $p_{\theta}=0$ (balancing) and its unique maximum when all phases are identical (synchronization). All other critical points of $U_{1}$ are isolated in the shape manifold $T^{N} / S^{1}$ and are saddle points of $U_{1}$.
The phase model $\dot{\boldsymbol{\theta}}=\mathbf{u}$ with the gradient control (5) forces convergence of all solutions to the critical set of $U_{1}$. If $K<0$, then only the set of synchronized states is asymptotically stable and every other equilibrium is unstable. If $K>0$, then only the balanced set where $p_{\theta}=0$ is asymptotically stable and every other equilibrium is unstable.

Proof: The gradient dynamics $\dot{\boldsymbol{\theta}}=-K \operatorname{grad} U_{1}$ forces convergence of all solutions to the set of critical points of $U_{1}$, characterized by the $N$ algebraic equations

$$
\left\langle p_{\theta}, i e^{i \theta_{k}}\right\rangle=0, \quad 1 \leq k \leq N
$$

Critical points where $p_{\theta}=0$ are global minima of $U_{1}$. As a consequence, the balanced set is asymptotically stable if $K>0$ and unstable if $K<0$. From (6), critical points where $p_{\theta}=$ $\left|p_{\theta}\right| e^{i \Psi} \neq 0$ are characterized by $\sin \left(\theta_{k}-\Psi\right)=0$, that is, $N-M$ phases synchronized at $\Psi \bmod 2 \pi$ and $M$ phases synchronized at $(\Psi+\pi) \bmod 2 \pi$, with $0 \leq M<(N / 2)$. At those points, $\left|p_{\theta}\right|=1-(2 M) /(N) \geq(1 / N)$. The value $M=0$ defines a synchronized state and corresponds to a global maximum of $U_{1}$. As a consequence, the set of synchronized states is asymptotically stable if $K<0$ and unstable if $K>0$.

Every other value $1 \leq M<(N / 2)$ corresponds to a saddle and is, therefore, unstable both for $K>0$ and $K<0$. For $\left|p_{\theta}\right|>(1 / N)$, this is because the second derivative

$$
\frac{\partial^{2} U_{1}}{\partial \theta_{k}^{2}}=\frac{1}{N}-\left\langle p_{\theta}, e^{i \theta_{k}}\right\rangle=\frac{1}{N}-\cos \left(\Psi-\theta_{k}\right)\left|p_{\theta}\right|
$$

takes negative values if $\theta_{k}=\Psi$ and positive values if $\theta_{k}=$ $\Psi+\pi$. As a consequence, a small variation $\delta \theta_{k}$ at those critical points decreases the value of $U_{1}$ if $\theta_{k}=\Psi$ and increases the value of $U_{1}$ if $\theta_{k}=\Psi+\pi$. For $\left|p_{\theta}\right|=(1 / N)$, a small variation of two angles can be used to prove the desired result.

The consequence of Theorem 1 is that parallel formations are stabilized by all-to-all sinusoidal coupling of the phases differences, i.e., the control law (5) with $K<0$. With $K>0$, the same control law stabilizes the center of mass of the particles to a fixed point. The fact that the remaining equilibria are saddles suggests that the conclusions of Theorem 1 are almost global, that is, almost all solutions either converge to the synchronized state $(K<0)$ or to the balanced set $(K>0)$.

We note that the conclusions of Theorem 1 can be equivalently stated in a rotating frame, that is, for the phase model

$$
\dot{\boldsymbol{\theta}}=\omega_{0} \mathbf{1}-K \operatorname{grad} U_{1}, \quad \omega_{0} \in \mathbb{R} .
$$

The convergence analysis is unchanged because of the property $\left\langle\operatorname{grad} U_{1}, \mathbf{1}\right\rangle=0$, which is a consequence of the invariance of $U_{1}$ under the action of the symmetry group $S^{1}$.

For $\omega_{0}=0$, the steady state of the phase model (8) gives rise to straight orbits in the particle model (1): synchronization then means parallel motion in a fixed direction, with arbitrary but constant relative spacing, which is a relative equilibrium of the model. In contrast, balancing means straight orbits towards or away from a fixed center of mass, which does not correspond to a relative equilibrium of the model. 


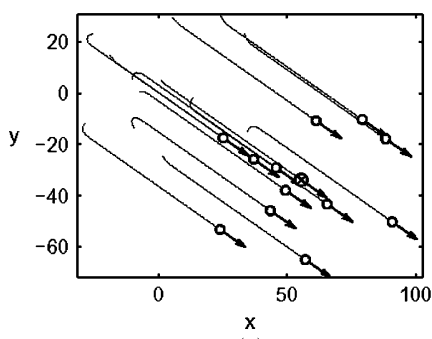

(a)

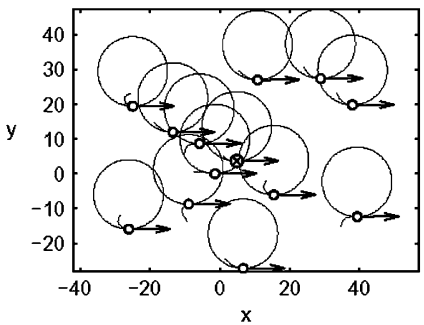

(c)

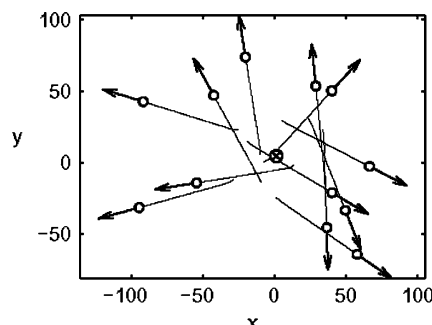

(b)

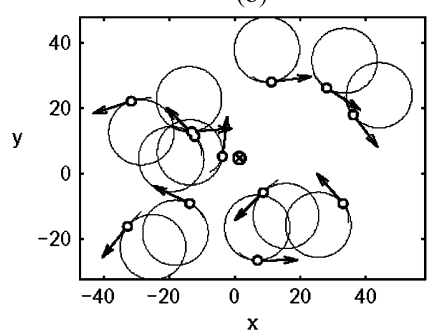

(d)
Fig. 1. Four different types of collective motion of $N=12$ particles obtained with the phase control (8). The position of each particle, $r_{k}$, and its time-derivative, $\dot{r}_{k}$, are illustrated by a circle with an arrow. The center of mass of the group, $R$, and its time-derivative, $R$, are illustrated by a crossed circle with an arrow. (a) $\omega_{0}=0$ and $K<0$. (b) $\omega_{0}=0$ and $K>0$. (c) $\omega_{0} \neq 0$ and $K<0$. (d) $\omega_{0} \neq 0$ and $K>0$. Only (a) is a relative equilibrium of the particle model (1).

For $\omega_{0} \neq 0$, the steady state of the phase model (8) gives rise to circular orbits of radius $\rho_{0}=\left|\omega_{0}\right|^{-1}$ in the particle model (1). In general, particles orbit different circles. Synchronization imposes parallel orientation of all velocity vectors whereas balancing imposes a fixed center of mass. A collective motion with fixed center of mass corresponds to a relative equilibrium of the model only if all particles orbit the same circle.

The four different types of collective motion associated with the phase model (8) are illustrated in Fig. 1.

\section{Stabilization OF CiRCUlar Formations}

In contrast to the phase control designed in the previous section, we now propose a spacing control that achieves global convergence to a circular relative equilibrium of the particle model (1).

We start our analysis with the observation that under the constant control $u_{k}=\omega_{0} \neq 0$, each particle travels at constant, unit speed on a circle of radius $\rho_{0}=\left|\omega_{0}\right|^{-1}$. The center of the circle traversed by particle $k$ is $c_{k}=r_{k}+i \omega_{0}^{-1} e^{i \theta_{k}}$. Multiplied by the constant factor $-i \omega_{0}, c_{k}$ becomes

$$
s_{k}=-i \omega_{0} c_{k}=e^{i \theta_{k}}-i \omega_{0} r_{k}
$$

A circular relative equilibrium is obtained when all the centers coincide; this corresponds to the algebraic condition

$$
P \mathbf{s}=0 \quad P=I_{N}-\frac{1}{N} \mathbf{1 1}^{\mathbf{T}}
$$

This suggests to choose a stabilizing control that minimizes the Lyapunov function

$$
S(\mathbf{r}, \boldsymbol{\theta})=\frac{1}{2}\|P \mathbf{s}\|^{2}
$$

Noting that

$$
\dot{s}_{k}=i e^{i \theta_{k}}\left(u_{k}-\omega_{0}\right)
$$

the time-derivative of $S$ along the solutions of (1) is

$$
\dot{S}=\langle P \mathbf{s}, P \dot{\mathbf{s}}\rangle=\sum_{k=1}^{N}\left\langle P_{k} \mathbf{s}, i e^{i \theta_{k}}\right\rangle\left(u_{k}-\omega_{0}\right),
$$

where $P_{k}$ denotes the $k$ th row of the matrix $P$ and where we have used the fact that $P$ is a projector, i.e., $P^{2}=P$. Choosing the control law

$$
u_{k}=\omega_{0}-\kappa<P_{k} \mathbf{s}, i e^{i \theta_{k}}>, \quad \kappa>0
$$

results in

$$
\dot{S}=-\kappa \sum_{k=1}^{N}\left\langle P_{k} \mathbf{s}, i e^{i \theta_{k}}\right\rangle^{2} \leq 0 .
$$

Noting that

$$
P_{k} \mathbf{s}=s_{k}-\frac{1}{N} \mathbf{1}^{\mathbf{T}} \mathbf{s}=e^{i \theta_{k}}-i \omega_{0} r_{k}-\left(\dot{R}-i \omega_{0} R\right)
$$

we obtain

$$
\begin{aligned}
\left\langle P_{k} \mathbf{s}, i e^{i \theta_{k}}\right\rangle & =-\left\langle\omega_{0}\left(r_{k}-R\right), e^{i \theta_{k}}\right\rangle-\left\langle\dot{R}, i e^{i \theta_{k}}\right\rangle \\
& =-\left\langle\omega_{0} \tilde{r}_{k}, e^{i \theta_{k}}\right\rangle-\frac{\partial U_{1}}{\partial \theta_{k}}
\end{aligned}
$$

where we denote by $\tilde{r}_{k}=r_{k}-R$ the relative position of particle $k$ from the group center of mass $R=\frac{1}{N} \sum_{k=1}^{N} r_{k}$. Using (16), we rewrite the control law (14) as

$$
u_{k}=\kappa \frac{\partial U_{1}}{\partial \theta_{k}}+\omega_{0}\left(1+\kappa\left\langle\tilde{r}_{k}, \dot{r}_{k}\right\rangle\right), \quad \kappa>0, \omega_{0} \neq 0
$$

Lyapunov analysis provides the following global convergence result.

Theorem 2: Consider the particle model (1) with the spacing control (17). All solutions converge to a relative equilibrium defined by a circular formation of radius $\rho_{0}=\left|\omega_{0}\right|^{-1}$ with direction determined by the sign of $\omega_{0} \neq 0$.

Proof: The Lyapunov function $S(\mathbf{r}, \boldsymbol{\theta})$ defined in (11) is positive definite and proper in the reduced shape space, that is, when all points $(\mathbf{r}, \boldsymbol{\theta})$ that differ only by a rigid translation $\mathbf{r}+\mathbf{1} r_{0}$ and a rigid rotation $\boldsymbol{\theta}+\mathbf{1} \theta_{0}$ are identified. From (15), $S$ 
is nonincreasing along the solutions and, by the LaSalle Invariance principle, solutions for the reduced system on shape space converge to the largest invariant set $\Lambda$ where

$$
\kappa\left\langle P_{k} \mathbf{s}, i e^{i \theta_{k}}\right\rangle \equiv 0
$$

for $k=1, \ldots, N$. In this set, $\dot{\theta}_{k}=\omega_{0}$ and $s_{k}$ is constant for all $k=1, \ldots N$. This means that (18) can hold only if $P \mathbf{s} \equiv 0$. As a result, $\mathbf{s}=1 s_{0}$ for some fixed $s_{0} \in \mathbb{C}$, i.e., all particles orbit the same circle of radius $\rho_{0}$.

\section{Phase Symmetry Breaking IN CIRCUlar Formations}

The spacing control law of Section IV stabilizes particle motions to a unique set in the physical plane modulo the symmetry group of rigid displacements. In contrast, the phase arrangement of the particles is arbitrary. To reduce the dimension of the equilibrium set, we combine the spacing potential $S(\mathbf{r}, \boldsymbol{\theta})$ defined in (11) with a phase potential $U(\boldsymbol{\theta})$ that is minimum at the desired phase configuration. We require that $U(\boldsymbol{\theta})$ preserves the $S^{1}$ symmetry of rigid rotation, that is, $\langle\operatorname{grad} U, \mathbf{1}\rangle=0$.

Theorem 3: Consider the particle model (1) and a smooth phase potential $U(\boldsymbol{\theta})$ that satisfies $\langle\operatorname{grad} U, \mathbf{1}\rangle=0$. The control law

$$
u_{k}=\omega_{0}\left(1+\kappa\left\langle\tilde{r}_{k}, \dot{r}_{k}\right\rangle\right)-\frac{\partial\left(U-\kappa U_{1}\right)}{\partial \theta_{k}}, \quad \omega_{0} \neq 0
$$

enforces convergence of all solutions to the set of relative equilibria defined by circular formations where all particles move around the same circle of radius $\rho_{0}$ and direction given by the sign of $\omega_{0}$ with a phase arrangement in the critical set of $U$. Every (local) minimum of $U$ defines an asymptotically stable set of relative equilibria. Every relative equilibrium where $U$ does not reach a minimum is unstable.

Proof: We use the composite Lyapunov function

$$
V(\mathbf{r}, \boldsymbol{\theta})=\kappa S(\mathbf{r}, \boldsymbol{\theta})+U(\boldsymbol{\theta})
$$

which is lower bounded since $S \geq 0$ and $U$ takes values in a compact set. The time-derivative of $V$ along the solutions of (1) is

$$
\dot{V}=\sum_{k=1}^{N}\left(\kappa\left\langle P_{k} \mathbf{s}, i e^{i \theta_{k}}\right\rangle\left(u_{k}-\omega_{0}\right)+\frac{\partial U}{\partial \theta_{k}} u_{k}\right)
$$

which, using the property $\left\langle\operatorname{grad} U, 1 \omega_{0}\right\rangle=0$, becomes

$$
\dot{V}=\sum_{k=1}^{N}\left(\kappa\left\langle P_{k} \mathbf{s}, i e^{i \theta_{k}}\right\rangle+\frac{\partial U}{\partial \theta_{k}}\right)\left(u_{k}-\omega_{0}\right) .
$$

Because the control (19) is

$$
u_{k}=\omega_{0}-\kappa\left\langle P_{k} \mathbf{s}, i e^{i \theta_{k}}\right\rangle-\frac{\partial U}{\partial \theta_{k}}
$$

the Lyapunov function $V$ satisfies $\dot{V}=-\sum_{k=1}^{N}\left(u_{k}-\omega_{0}\right)^{2} \leq 0$ along the closed-loop solutions. By the LaSalle Invariance principle, solutions for the reduced system on shape space converge to the largest invariant set $\Lambda$ where

$$
\kappa\left\langle P_{k} \mathbf{s}, i e^{i \theta_{k}}\right\rangle=-\frac{\partial U}{\partial \theta_{k}}
$$

for $k=1, \ldots, N$. In the set $\Lambda$, the dynamics reduce to $\dot{\theta}_{k}=$ $\omega_{0}$, which implies that $U$ is constant. Therefore, the right-hand side of (24) vanishes in the set $\Lambda$, which implies $P \mathbf{s}=0$ since $\dot{\mathbf{s}}=0$. We conclude that solutions converge to a circular relative equilibrium and that the asymptotic phase arrangement is in the critical set of $U$.

Consider the set $E$ of circular relative equilibria of radius $\rho_{0}$ with a phase configuration in the critical set of $U$. Because $V(\mathbf{r}, \boldsymbol{\theta})=U(\boldsymbol{\theta})$ in $E$, local minima of $U$ correspond to local minima of the Lyapunov function. Any connected subset of $E$ on which $U$ reaches a strict minimum is, therefore, asymptotically stable. Note that because $E$ is a set of equilibria, $U$ is constant on any connected subset. In contrast, consider $\overline{\mathbf{x}}=(\overline{\mathbf{r}}, \overline{\boldsymbol{\theta}}) \in$ $E$ such that $U(\overline{\boldsymbol{\theta}})$ is not a minimum and denote by $E_{\overline{\mathbf{x}}}$ the connected component of $E$ containing $\overline{\mathbf{x}}$. $U$ is constant in $E_{\overline{\mathbf{x}}}$. To show instability of $\overline{\mathbf{x}}$, consider a compact neighborhood $B(\overline{\mathbf{x}})$ $B(\overline{\mathbf{x}}) \in E_{\overline{\mathbf{x}}}$ contains no other relative equilibrium. A solution with initial condition in $B(\overline{\mathbf{x}})$ either asymptotically converges to $E_{\overline{\mathbf{x}}}$ or leaves $B(\overline{\mathbf{x}})$ after a finite time. Let $\mathbf{x}=(\overline{\mathbf{r}}, \boldsymbol{\theta}) \in B(\overline{\mathbf{x}})$ such that $U(\boldsymbol{\theta})<U(\overline{\boldsymbol{\theta}})$. Then the solution with initial condition $\mathbf{x}$ cannot converge to $E_{\overline{\mathbf{x}}}$ (and therefore leaves $B$ after a finite time) since $V$ decreases along solutions and $V(\overline{\mathbf{r}}, \boldsymbol{\theta})<V(\overline{\mathbf{r}}, \overline{\boldsymbol{\theta}})$. Because $V(\overline{\mathbf{x}})$ is not minimum, $\mathbf{x}$ can be chosen arbitrary close to $\overline{\mathbf{x}}$, which proves instability of $\overline{\mathbf{x}}$.

Theorem 3 thus provides a global convergence analysis of closed-loop dynamics achieved with the control law (19). It shows that solutions either converge to circular relative equilibria with a phase configuration that (locally) minimizes the phase potential $U$ or belong to the stable manifold of an unstable equilibrium. As an illustration, simulation results in Fig. 2 illustrate the convergence result for the choice $U=K U_{1}$. For $K=0$, the control law (19) achieves convergence to a circular formation but the asymptotic phase arrangement is not constrained. For $K<0$, the control law (19) forces convergence to the synchronized circular formation. For $K>0$, the control law (19) forces convergence to a balanced circular formation. Note that for $K=\kappa$, the expression of the control law simplifies to

$$
u_{k}=\omega_{0}\left(1+\kappa\left\langle\tilde{r}_{k}, \dot{r}_{k}\right\rangle\right) .
$$

The stability analysis in Theorem 3 is entirely determined by the critical points of the phase potential $U$ : (local) minima correspond to stable equilibria and other critical points correspond to unstable relative equilibria. When a critical point is nondegenerate, this analysis extends to the Jacobian linearization of the closed-loop system, providing (local) exponential stability conclusions. We say that a critical point $\overline{\boldsymbol{\theta}}$ is nondegenerate (in 


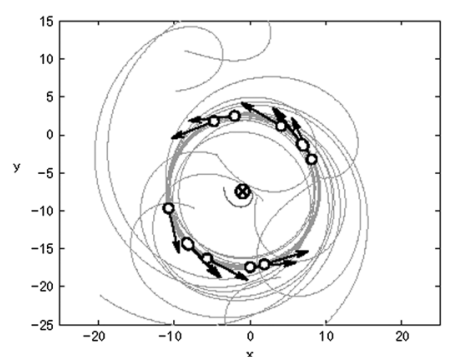

(a)

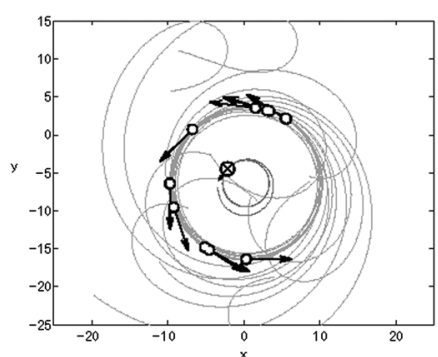

(b)

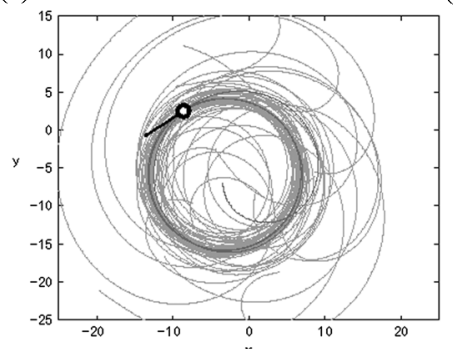

(c)

Fig. 2. Circular formations achieved with the control (19) with $N=12, \omega_{0}=$ $\kappa=0.1$, and $U=K U_{1}$. (a) Convergence to a balanced circular formation $(K>0)$. (b) Convergence to a circular formation with no constraint on the asymptotic phase arrangement $(K=0)$. (c) Convergence to a synchronized circular formation $(K<0)$.

$\left.T^{N} / S^{1}\right)$ if all eigenvalues of the Hessian $\left(\partial^{2} U\right) /\left(\partial \theta^{2}\right)(\overline{\boldsymbol{\theta}})$ are different from zero, except for the zero eigenvalue (with eigenvector 1) associated to the rotational symmetry.

Theorem 4: Consider as in Theorem 3 the particle model (1), a smooth phase potential $U(\boldsymbol{\theta})$ that satisfies $\langle\operatorname{grad} U, \mathbf{1}\rangle=0$, and the control law (19). A relative equilibrium determined by a nondegenerate critical point $\bar{\theta}$ of $U$ is exponentially stable if $\overline{\boldsymbol{\theta}}$ is a (local) minimum and exponentially unstable otherwise.

Proof: The proof is a local version of the proof of Theorem 3 around the relative equilibrium $\overline{\mathbf{x}}=(\overline{\mathbf{r}}, \overline{\boldsymbol{\theta}})$. In the coordinates $(\mathbf{s}, \boldsymbol{\theta})$, the quadratic approximation of $V$ at $\overline{\mathbf{x}}$ is

$$
\delta V=\frac{1}{2}\left(\kappa\langle P \mathbf{s}, P \mathbf{s}\rangle+\delta \boldsymbol{\theta}^{T} H \delta \boldsymbol{\theta}\right)
$$

where $\delta \boldsymbol{\theta}=\boldsymbol{\theta}-\left(\overline{\boldsymbol{\theta}}+\omega_{0} t \mathbf{1}\right)$ and $H=\left(\partial^{2} U\right) /\left(\partial \boldsymbol{\theta}^{2}\right)(\overline{\boldsymbol{\theta}})$. Likewise, the control law (23) linearizes to

$$
\delta u_{k}=-\kappa\left\langle P_{k} \mathbf{s}, i e^{i\left(\bar{\theta}_{k}+\omega_{0} t\right)}\right\rangle-H_{k} \delta \boldsymbol{\theta}
$$

where $H_{k}$ is the $k$ th row of $H$. Then

$$
\begin{aligned}
\delta \dot{V} & =\kappa \sum_{k=1}^{N}\left\langle P_{k} \mathbf{s}, i e^{i\left(\bar{\theta}_{k}+\omega_{0} t\right)}\right\rangle \delta u_{k}+(H \delta \boldsymbol{\theta})^{T} \delta \mathbf{u} \\
& =\sum_{k=1}^{N}\left(\kappa\left\langle P_{k} \mathbf{s}, i e^{i\left(\bar{\theta}_{k}+\omega_{0} t\right)}\right\rangle+H_{k} \delta \boldsymbol{\theta}\right) \delta u_{k} \\
& =-\sum_{k=1}^{N}\left(\delta u_{k}\right)^{2} \leq 0
\end{aligned}
$$

along the closed-loop solutions of the linearized system.
The stability analysis then proceeds as in the proof of Theorem 3: if $\overline{\boldsymbol{\theta}}$ is a minimum, then $H \geq 0$ and the LaSalle invariance principle proves asymptotic stability of the linearized (periodic) closed-loop system, which implies exponential stability of the equilibrium. In contrast, if $\overline{\boldsymbol{\theta}}$ is not a minimum, then there exist initial conditions for which $\delta V<0$. The corresponding solutions of the linearized system cannot converge to the equilibrium and must diverge exponentially.

The above result yields a systematic and general design methodology by reducing the design of exponentially stabilizing control laws to the construction of phase potentials. Specifically, control laws that exponentially stabilize isolated relative circular equilibria are automatically derived from phase potentials $U$ that have nondegenerate minima at the desired location. The phase potential $U_{1}$ of (4) achieves this objective for the stabilization of the synchronized circular formation. The next section focuses on the construction of more general phase potentials that can be used to isolate specific balanced circular formations.

\section{EXPONENTIAL STABILIZATION OF ISOLATED CIRCULAR RELATIVE EQUILIBRIA}

\section{A. Stabilization of Higher Momenta}

When the phase potential $U_{1}$ reaches its minimum, the phase arrangement of the particles is only stabilized to the balanced set, which is high dimensional. More general phase potentials are introduced in this section in order to reduce the dimension of this equilibrium set. A natural generalization of the potential $U_{1}$ is a potential

$$
U_{m}=\frac{N}{2}\left|p_{m \theta}\right|^{2},
$$

which depends on the $m$ th moment $p_{m \theta}$ of the phase distribution on the circle, defined as

$$
p_{m \theta}=\frac{1}{m N} \sum_{k=1}^{N} e^{i m \theta_{k}}, \quad m=1,2, \ldots .
$$

Note that $p_{1 \theta}=p_{\theta}$. The next proposition is a direct generalization of Theorem 1.

Theorem 5: Let $m \in \mathbb{N}$. The potential $U_{m}=(N / 2)\left|p_{m \theta}\right|^{2}$ reaches its unique minimum when $p_{m \theta}=0$ (balancing modulo $(2 \pi) /(m)$ and its unique maximum when the phase difference between any two phases is an integer multiple of $(2 \pi) /(m)$ (synchronization modulo $(2 \pi) /(m)$ ). All other critical points of $U_{m}$ are isolated in the shape manifold $T^{N} / S^{1}$ and are saddle points of $U_{m}$.

Proof: Critical points of $U_{m}$ are the roots of

$$
\frac{\partial U_{m}}{\partial \theta_{k}}=\left\langle p_{m \theta}, i e^{i m \theta_{k}}\right\rangle=0, \quad k=1, \ldots, N .
$$

Critical points for which $p_{m \theta}=0$ are global minima of $U_{m}$. Critical points for which $p_{m \theta}=\left|p_{m \theta}\right| e^{i \Psi_{m}} \neq 0$ are characterized by $N-M$ phases satisfying $m \theta_{k}=\Psi_{m} \bmod 2 \pi$ and $M$ phases satisfying $m \theta_{k}=\left(\Psi_{m}+\pi\right) \bmod 2 \pi$, with $0 \leq M<$ $(N / 2)$. At those critical points, $m\left|p_{m \theta}\right|=1-(2 M) /(N) \geq$ 


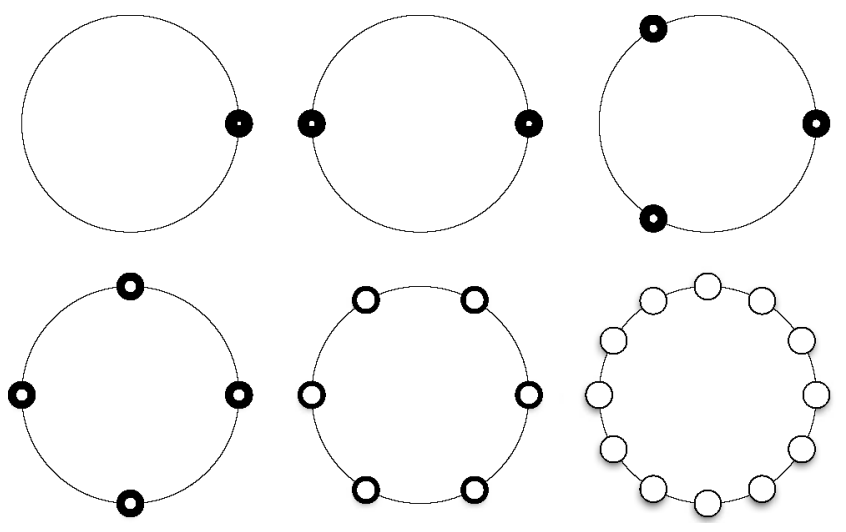

Fig. 3. Six possible different symmetric patterns for $N=12$ corresponding to $M=1,2,3,4,6$ and 12 . The top left is the synchronized state and the bottom right is the splay state. The number of collocated phases is illustrated by the width of the black annulus denoting each phase cluster.

$(1 / N) . U_{m}$ is maximized when $M=0$. Critical points for which $1 \leq M<(N / 2)$ and $m\left|p_{m} \theta\right|>(1 / N)$ are saddles because the second derivative

$$
\begin{aligned}
\left(\partial^{2} U_{m}\right) /\left(\partial \theta_{k}^{2}\right) & =\frac{1}{N}-m\left\langle p_{m \theta}, e^{i m \theta_{k}}\right\rangle \\
& =\frac{1}{N}-\cos \left(\Psi_{m}-m \theta_{k}\right) m\left|p_{m \theta}\right|
\end{aligned}
$$

takes negative values if $m \theta_{k}=\Psi_{m}$ and positive values if $m \theta_{k}=\Psi_{m}+\pi$. As a consequence, a small variation $\delta \theta_{k}$ at those critical points decreases the value of $U_{m}$ if $m \theta_{k}=\Psi_{m}$ and increases the value of $U_{m}$ if $m \theta_{k}=\Psi_{m}+\pi$. For $\left|p_{m} \theta\right|=(1 / N)$, a small variation of two angles can be used to prove the desired result.

We show in the next section how linear combinations of the potentials $U_{m}$ enable the stabilization of specific sets of isolated relative equilibria characterized by various discrete symmetry groups.

\section{B. Symmetric Balanced Patterns}

Let $1 \leq M \leq N$ be a divisor of $N$. An $(M, N)$-pattern is a symmetric arrangement of $N$ phases consisting of $M$ clusters uniformly spaced around the unit circle, each with $N / M$ synchronized phases. For any $N$, there exist at least two symmetric patterns: the $(1, N)$-pattern, which is the synchronized state, and the $(N, N)$-pattern, which is the splay state, characterized by $N$ phases uniformly spaced around the circle. Fig. 3 provides an illustration of all symmetric balanced patterns for $N=12$.

Symmetric balanced patterns are extremals of the potentials $U_{m}$. As a consequence, they are characterized as minimizers of well-chosen potentials; these can be written as linear combinations of the $U_{m}$. This result for $M=N$, i.e., the splay state, was also presented in [27]. We first prove a technical lemma that we will use in making this characterization explicit.

Lemma 1: Consider the following sum where $m, M \in \mathbb{N}$

$$
P_{m}^{(M)} \triangleq \sum_{j=1}^{M} e^{i \frac{2 \pi m}{M} j}
$$

If $(m / M) \in \mathbb{N}$, then $P_{m}^{(M)}=M$, otherwise $P_{m}^{(M)}=0$.

Proof: If $(m / M) \in \mathbb{N}$ then $\left.e^{i(2 \pi m / M} j\right)=1$ for all $j \in \mathbb{N}$ which proves the first part of the lemma. To prove the second part, we treat (29) as the sum of a geometric series and evaluate it for all $m \in \mathbb{N}$ that satisfy $(m / M) \notin \mathbb{N}$. Multiplying both sides of (29) by $e^{i(2 \pi m / M)}$ gives

$$
P_{m}^{(M)} e^{i \frac{2 \pi m}{M}}=\sum_{j=1}^{M} e^{i \frac{2 \pi m}{M}(j+1)}=P_{m}^{(M)}-e^{i \frac{2 \pi m}{M}}+e^{i \frac{2 \pi m}{M}(M+1)} .
$$

Rearranging terms and solving for $P_{m}^{(M)}$ yields

$$
P_{m}^{(M)}=e^{i \frac{2 \pi m}{M}} \frac{e^{i 2 \pi m}-1}{e^{i \frac{2 \pi m}{M}}-1}
$$

which shows that $P_{m}^{(M)}=0$ since the numerator of (30) vanishes for all $m \in \mathbb{N}$ that satisfy $(m / M) \notin \mathbb{N}$.

Theorem 6: Let $1 \leq M \leq N$ be a divisor of $N$. Then $\boldsymbol{\theta} \in T^{N}$ is an $(M, N)$-pattern if and only if it is a global minimum of the potential

$$
U^{M, N}=\sum_{m=1}^{M} K_{m} U_{m}
$$

with $K_{m}>0$ for $m=1, \ldots, M-1$ and $K_{M}<0$.

Proof: The global minimum of $U^{M, N}$ is reached (only) when $K_{m} U_{m}$ is minimized for each $m$. If $K_{m}>0$ for $m=1, \ldots, M-1$ and $K_{M}<0$, following (25) and (26) this means the global minimum corresponds to $p_{m \theta}=0$ for $m=1, \ldots, M-1$ and $p_{M \theta}=(1 / M)$. We show that this implies an $(M, N)$-pattern configuration. From the condition $p_{M \theta}=(1 / M)$ and Theorem 5, we can conclude that there are $M$ clusters such that the $k$ th cluster is of size $N_{k} \geq 0$ at phase $\Theta_{k}=(2 \pi) /(M) k, k=1, \ldots, M$, where $\sum_{k=1}^{M} N_{k}=N$. We would like to show that $N_{k}=(N / M) \in \mathbb{N}$ for all $k=1, \ldots, M$. We have

$$
p_{m \theta}=\frac{1}{m N} \sum_{k=1}^{M} N_{k} e^{i \frac{2 \pi m}{M} k}=0, \quad m=1, \ldots, M-1
$$

and

$$
M p_{M \theta}=\frac{1}{N} \sum_{k=1}^{M} N_{k} e^{i 2 \pi k}=1 .
$$

Equations (32) and (33) are a system of linear equations in the unknown variables $\mathbf{x}=\left(N_{1}, \ldots, N_{M}\right)^{T}$. Namely, (32) and (33) can be written as $A \mathbf{x}=\mathbf{b}$ where $A=A^{T} \in \mathbb{C}^{M \times M}$ with $[A]_{k j}=e^{i(2 \pi / M) k j}, j, k=1, \ldots, M$ and $\mathbf{b}=(0, \ldots, 0, N)^{T} \in \mathbb{R}^{M}$. The inverse of $A$ is given by $A^{-1}=(1 / M) \bar{A}^{T}$, where the bar denotes the complex conjugate. To see this, observe that

$$
\left[A \bar{A}^{T}\right]_{k j}=\sum_{l=1}^{M} e^{i \frac{2 \pi(k-j)}{M} l}, \quad j, k=1, \ldots, M .
$$

For $j=k$, (34) evaluates to $M$ by Lemma 1 . For $j \neq k$, we have $|k-j|<M$ and (34) evaluates to zero by Lemma 1 . Therefore, the solution to the system of (32) and (33) is $\mathbf{x}=(1 / M) \bar{A}^{T} \mathbf{b}$. 
Since both the $M$ th row and column of $\bar{A}^{T}$ are all ones, we find that $N_{k}=(N / M)$ for all $k=1, \ldots, M$.

Next, we show that an $(M, N)$-pattern configuration minimizes each $K_{m} U_{m}$ for $m=1, \ldots, M$. For an $(M, N)$-pattern, the size of cluster $k$ is $N / M$ and its phase is given by $\Theta_{k}=(2 \pi) /(M) k$, where $k=1, \ldots, M$. Recall that the $m$ th moment $p_{m \theta}$ of the phase distribution is given by (26). Evaluated at an $(M, N)$-pattern, the $m$ th moment becomes

$$
\begin{aligned}
p_{m \theta}^{(M)} & =\frac{1}{N m} \frac{N}{M} \sum_{j=1}^{M} e^{i m \Theta_{j}} \\
& =\frac{1}{M m} \sum_{j=1}^{M} e^{i \frac{2 \pi m}{M} j}, \quad m=1, \ldots, M .
\end{aligned}
$$

By Lemma $1, p_{m \theta}^{(M)}=(1 / m)$ for $(m / M) \in \mathbb{N}$ and zero otherwise. Therefore, for phases in an $(M, N)$-pattern, $p_{m \theta}=0$ for $m=1, \ldots, M-1$ and $p_{M \theta}=(1 / M)$.

Corollary 1: Let $M=N$. Then $\boldsymbol{\theta} \in T^{N}$ is an $(N, N)$-pattern, i.e., the splay state, if and only if it is a global minimum of the potential

$$
U^{N, N}=\sum_{m=1}^{\left\lfloor\frac{N}{2}\right\rfloor} K_{m} U_{m}
$$

with $K_{m}>0$ for $m=1, \ldots,\lfloor(N / 2)\rfloor$, where $\lfloor(N / 2)\rfloor$ is the largest integer less than or equal to $(N / 2)$.

Proof: By Theorem 6, the phases are in the splay state if and only if they correspond to the global minimum of the potential (31) with $M=N$. Since $p_{m \theta}=0$ imposes two constraints on the system for each $m$, minimizing the potential $U^{M, N}$ imposes $2 N$ constraints on the phase arrangement. However, for potentials with $S^{1}$ symmetry, the dimension of the shape space is $N-1$. Therefore, we need only set $p_{m \theta}=0$ for $m=1, \ldots,\lfloor(N / 2)\rfloor$ since that imposes $2\lfloor(N / 2)\rfloor \geq N-1$ independent constraints.

Due to the characterization of Theorem 6, stabilizing control laws for $(M, N)$-pattern circular formations are directly and systematically provided by Theorem 3 .

Theorem 7: Each $(M, N)$-pattern circular formation of radius $\rho_{0}$ is an isolated relative equilibrium of the particle model (1) and is exponentially stabilized by the control law

$$
u_{k}=\omega_{0}\left(1+\kappa\left\langle\tilde{r}_{k}, \dot{r}_{k}\right\rangle\right)-\frac{\partial\left(U^{M, N}-\kappa U_{1}\right)}{\partial \theta_{k}} .
$$

Proof: The theorem is a consequence of Theorems 3, 4, and 6. We only need to prove that each $(M, N)$-pattern defines a nondegenerate critical point of the potential $U^{(M, N)}$.

Let the negative gradient of the potential $U^{M, N}$ be defined in terms of the coupling function, $\Gamma\left(\theta_{k j}\right)$, i.e.,

$$
-\frac{\partial U^{M, N}}{\partial \theta_{k}}=\sum_{j=1}^{N} \sum_{m=1}^{M} \frac{K_{m}}{m} \sin m \theta_{k j} \triangleq \sum_{j=1}^{N} \Gamma\left(\theta_{k j}\right)
$$

where $K_{m}>0$ for $m=1, \ldots, M-1$ and $K_{M}<0$. Also, let $\Gamma^{\prime}\left(\theta_{k j}\right)$ be the derivative of $\Gamma\left(\theta_{k j}\right)$ with respect to $\theta_{k j}$, given by

$$
\Gamma^{\prime}\left(\theta_{k j}\right)=\sum_{m=1}^{M} K_{m} \cos m \theta_{k j} .
$$

As shown in [28], the linearization of coupling functions of this form about an $(M, N)$-pattern has $N$ eigenvalues that can be described as the union of two sets. The first set consists of the eigenvalue $\tilde{\lambda}^{(M)}$ with multiplicity $N-M$. These eigenvalues are associated with intra-cluster fluctuation. The second set consists of $M$ eigenvalues $\lambda_{p}^{(M)}, p=0, \ldots, M-1$. These eigenvalues are associated with intercluster fluctuation.

Both sets of eigenvalues can be expressed as functions of the Fourier coefficients of $\Gamma^{\prime}\left(\theta_{k j}\right)$. For a general coupling function, the Fourier expansion of $\Gamma^{\prime}\left(\theta_{k j}\right)$ is

$$
\Gamma^{\prime}\left(\theta_{k j}\right)=\sum_{l=1}^{\infty}\left(a_{l}^{\prime} \cos l \theta_{k j}+b_{l}^{\prime} \sin l \theta_{k j}\right) .
$$

The formulas for calculating the (real part of) the eigenvalues are as follows [28]:

$$
\begin{aligned}
\tilde{\lambda}^{(M)} & =\sum_{l=1}^{\infty} a_{M l}^{\prime} \\
\operatorname{Re}\left\{\lambda_{p}^{(M)}\right\} & =\sum_{l=1}^{\infty}\left(a_{M l}^{\prime}-\frac{a_{M(l-1)+p}^{\prime}+a_{M l-p}^{\prime}}{2}\right) .
\end{aligned}
$$

Note that only the $a_{l}^{\prime}$ coefficients determine stability and that $\operatorname{Re}\left\{\lambda_{p}^{(M)}\right\}=\operatorname{Re}\left\{\lambda_{M-p}^{(M)}\right\}$.

The $a_{l}^{\prime}$ coefficients are given by integrating

$$
a_{l}^{\prime}=\frac{1}{\pi} \int_{-\pi}^{\pi} \Gamma^{\prime}\left(\theta_{k j}\right) \cos l \theta_{k j} d \theta_{k j}
$$

which gives

$$
\begin{array}{ll}
a_{l}^{\prime}=K_{l}, & l=1, \ldots, M \\
& \text { and } \\
a_{l}^{\prime}=0, & l=0 \quad \text { or } l>M .
\end{array}
$$

As a result, $a_{M l}^{\prime}=0$ for $l>1$, which, using (41), yields $\tilde{\lambda}^{(M)}=K_{M}<0$. In addition, using (42), we find that $\lambda_{p}^{(M)}=$ $K_{M}-\left(K_{p}+K_{M-p}\right) /(2)<0$ for $p=1, \ldots, M-1$ and $\lambda_{0}^{(M)}=0$. The zero eigenvalue corresponds to rigid rotation of all $N$ phases [28]. Since the coupling function (38) is the gradient of the potential $U^{M, N}$, its Jacobian is the Hessian of $U^{M, N}$ and, consequently, all the eigenvalues are real. Therefore, each $(M, N)$-pattern defines a nondegenerate critical point of the potential $U^{M, N}$ since the Hessian has rank $N-1$.

Theorem 7 does not exclude convergence to circular formations that correspond to other critical points of the phase potential $U^{(M, N)}$. However, no other local minima were identified and simulations suggest large regions of attraction of the 


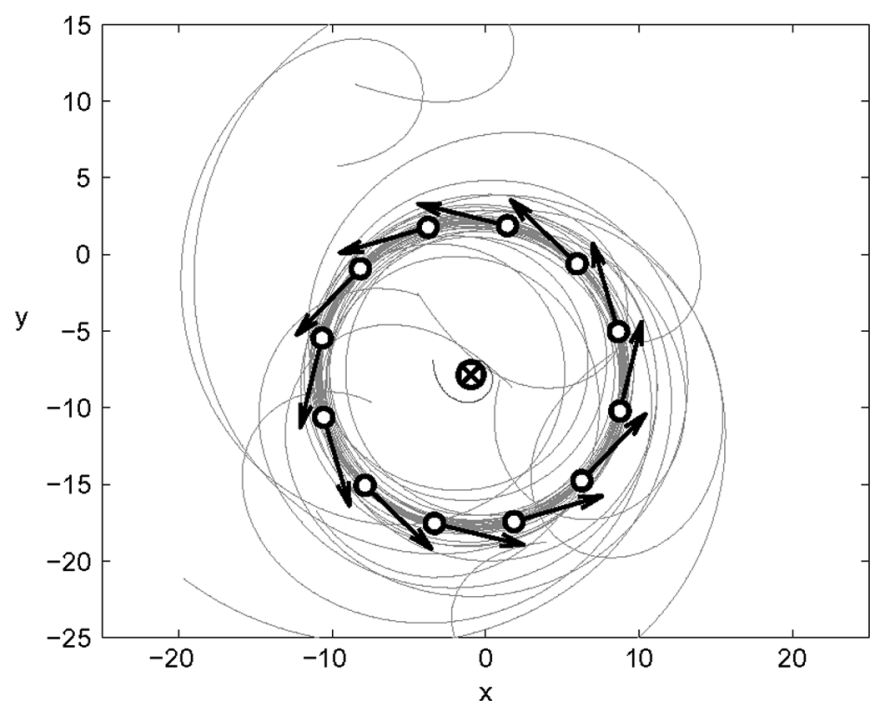

Fig. 4. Result of a numerical simulation starting from random initial conditions and stabilizing the splay state formation using the control (37) with $M=N=$ $12, \omega_{0}=\kappa=0.1, K_{m}=\omega_{0}$ for $m=1, \ldots,\lfloor(N / 2)\rfloor$, and $K_{m}=0$ for $m>\lfloor(N / 2)\rfloor$.

$(M, N)$-pattern circular formations. Fig. 4 illustrates a simulation of the splay state stabilization for $N=12$ particles using the control law (37).

\section{SPATIAL SyMmetry BREAKING IN PARALLEL FORMATIONS}

As shown in Theorem 1, the phase control $\mathbf{u}=\operatorname{grad} U_{1}$ suffices to stabilize parallel equilibria. However, the asymptotic relative positions of particles are arbitrary. In this section, we show how to reduce the dimension of the equilibrium set in close analogy to the design of the spacing control in Section IV.

We choose an arbitrary isolated parallel relative equilibrium in the shape space by imposing on the relative position $\tilde{r}_{k}$ of particle $k$ with respect to the center of mass a fixed length $\bar{\rho}_{k}$ and a fixed orientation $\bar{\psi}_{k}$ relative to the group direction. Assuming that all phases are synchronized at the relative equilibrium, this gives in complex notation

$$
\tilde{r}_{k}=r_{k}-R=\bar{\rho}_{k} e^{i\left(\theta_{k}-\bar{\psi}_{k}\right)} \triangleq d_{k}^{-1} e^{i \theta_{k}}
$$

where $d_{k}=\left(e^{i \bar{\psi}_{k}}\right) /\left(\bar{\rho}_{k}\right)$ is a constant complex number. The relative positions with respect to the center of mass must balance, that is, $\sum \tilde{r}_{k}=0$, which imposes the constraint

$$
\sum_{k=1}^{n} d_{k}^{-1}=0
$$

Motivated by the derivation of the spacing control in Section IV, we observe that the definition

$$
t_{k}=\frac{d_{k}}{1+d_{k}}\left(\tilde{r}_{k}+e^{i \theta_{k}}\right)
$$

allows one to specify the parallel relative equilibrium of interest by the conditions

$$
P \mathbf{t}=0 \quad\left|p_{\theta}\right|=1 \quad P=I_{N}-\frac{1}{N} \mathbf{1 1}^{T} .
$$

Indeed, the condition $\left|p_{\theta}\right|=1$ imposes phase synchronization, that is, $\boldsymbol{\theta}=\mathbf{1} \theta_{0}$ for some $\theta_{0} \in S^{1}$, whereas the condition $P \mathbf{t}=0$ implies $\mathbf{t}=1 t_{0}$ for some $t_{0} \in \mathbb{C}$, which in turn gives $\tilde{r}_{k}+e^{i \theta_{0}}=\left(1+d_{k}^{-1}\right) t_{0}$. Summing over $k$ yields $t_{0}=e^{i \theta_{0}}$, which corresponds to the desired relative equilibrium.

The desired relative equilibrium thus minimizes the Lyapunov function

$$
V(\mathbf{r}, \boldsymbol{\theta})=\kappa\left(\frac{1}{2}\|P \mathbf{t}\|^{2}-U_{1}(\boldsymbol{\theta})\right), \quad \kappa>0
$$

which, in analogy with the results of Section IV, suggests the control law

$$
u_{k}=(1+\kappa) \frac{\partial U_{1}}{\partial \theta_{k}}-\kappa\left\langle P_{k} \mathbf{t}, \frac{d_{k}}{d_{k}+1} i e^{i \theta_{k}}\right\rangle, \quad \kappa>0 .
$$

Lyapunov analysis provides the following result.

Theorem 8: Consider the particle model (1) with the control law (50). The parallel relative equilibrium defined by (48) is Lyapunov stable and a global minimum of the Lyapunov function (49). Moreover, for every $\kappa>0$, there exists an invariant set in which the Lyapunov function is nonincreasing along the solutions. In this set, solutions converge to a parallel relative equilibrium that satisfies

$$
\left\langle P_{k} \mathbf{t}, \frac{d_{k}}{d_{k}+1} i e^{i \theta_{0}}\right\rangle
$$

for some $\theta_{0} \in S^{1}$ and for $k=1, \ldots, N$.

Proof: In the coordinates $(\mathbf{t}, \boldsymbol{\theta})$, the system (1) becomes

$$
\begin{aligned}
& \dot{t}_{k}=\frac{d_{k}}{d_{k}+1}\left(P_{k} \boldsymbol{e}^{i \boldsymbol{\theta}}+i e^{i \theta_{k}} u_{k}\right) \\
& \dot{\theta}_{k}=u_{k} .
\end{aligned}
$$

Writing the control law (50) as $u_{k}=\left(\partial U_{1}\right) /\left(\partial \theta_{k}\right)+v_{k}=$ $-\left\langle P_{k} e^{i \boldsymbol{\theta}}, i e^{i \theta_{k}}\right\rangle+v_{k}$, and using the identity

$$
P_{k} \boldsymbol{e}^{\boldsymbol{i} \boldsymbol{\theta}}-i e^{i \theta_{k}}\left\langle P_{k} \boldsymbol{e}^{\boldsymbol{i} \boldsymbol{\theta}}, i e^{i \theta_{k}}\right\rangle=\left\langle P_{k} \boldsymbol{e}^{\boldsymbol{i} \boldsymbol{\theta}}, \boldsymbol{e}^{\boldsymbol{i} \boldsymbol{\theta}_{\boldsymbol{k}}}\right\rangle e^{i \theta_{k}}
$$

one obtains

$$
\begin{aligned}
\dot{t}_{k} & =\frac{d_{k}}{d_{k}+1}\left(\left\langle P_{k} \boldsymbol{e}^{i \boldsymbol{\theta}}, e^{i \theta_{k}}\right\rangle e^{i \theta_{k}}+i e^{i \theta_{k}} v_{k}\right) \\
\dot{\theta}_{k} & =\frac{\partial U_{1}}{\partial \theta_{k}}+v_{k} .
\end{aligned}
$$

Setting $v_{k}=0$, the time-derivative of the Lyapunov function (49) along solutions of (53) is

$$
\begin{aligned}
\left.\dot{V}\right|_{\mathbf{v}=0}=-\kappa & \left\|\operatorname{grad} U_{1}\right\|^{2} \\
& +\kappa \sum_{k=1}^{N}\left\langle P_{k} \mathbf{t}, \frac{d_{k}}{d_{k}+1} e^{i \theta_{k}}\right\rangle\left\langle P_{k} e^{i \boldsymbol{\theta}}, e^{i \theta_{k}}\right\rangle .
\end{aligned}
$$

Fix an arbitrary compact neighborhood $\mathcal{N}$ of the parallel equilibrium (46) that contains no critical point of $U_{1}$ other than the 
synchronized state. Then there exists a constant $c_{1}>0$ such that the inequality

$$
\left|\left\langle P_{k} \mathbf{t}, \frac{d_{k}}{d_{k}+1} e^{i \theta_{k}}\right\rangle\right| \leq c_{1} \| P \mathbf{t}
$$

holds in $\mathcal{N}$ for $k=1, \ldots, N$. We show that a similar constant $c_{2}>0$ exists for the inequality

$$
\left|\left\langle P_{k} e^{i \boldsymbol{\theta}}, e^{i \theta_{k}}\right\rangle\right| \leq c_{2}\left\|\operatorname{grad} U_{1}\right\|^{2} .
$$

Because the set $\mathcal{N}$ is compact, it is sufficient to establish (56) in the vicinity of critical points of $U_{1}$, that is when the right hand side of (56) vanishes. By assumption, the synchronized state is the only critical point of $U_{1}$ in the set $\mathcal{N}$. Locally around that state, we write $\left(\partial U_{1}\right) /\left(\partial \theta_{j}\right)=\theta_{j}-\theta_{a v}+$ h.o.t. where $\theta_{a v}=$ $(1 / N) \sum_{i=1}^{N} \theta_{i}$ and h.o.t. stands for "higher order terms." We then obtain

$$
\left\|\operatorname{grad} U_{1}\right\|^{2}=\sum_{j=1}^{N}\left(\theta_{j}-\theta_{a v}\right)^{2}+\text { h.o.t. }
$$

The left-hand side of (56) rewrites as

$$
\begin{aligned}
\left|1-\frac{1}{N} \sum_{j=1}^{N} \cos \left(\theta_{j}-\theta_{k}\right)\right| & \\
& =\frac{1}{2 N} \sum_{j=1}^{N}\left(\theta_{j}-\theta_{k}\right)^{2}+\text { h.o.t. }
\end{aligned}
$$

Using $\theta_{j}-\theta_{k}=\theta_{j}-\theta_{a v}+\theta_{a v}-\theta_{k}$ and the triangle inequality yield

$$
\begin{aligned}
& \frac{1}{2 N} \sum_{j=1}^{N}\left(\theta_{j}-\theta_{k}\right)^{2} \\
& \leq \frac{1}{N} \sum_{j=1}^{N}\left(\theta_{j}-\theta_{a v}\right)^{2}+\left(\theta_{k}-\theta_{a v}\right)^{2} \\
& \quad \leq \frac{N+1}{N} \sum_{j=1}^{N}\left(\theta_{j}-\theta_{a v}\right)^{2}
\end{aligned}
$$

which, from (58), provides the inequality

$$
\begin{aligned}
\left|1-\frac{1}{N} \sum_{j=1}^{N} \cos \left(\theta_{j}-\theta_{k}\right)\right| \\
\leq \frac{N+1}{N} \sum_{j=1}^{N}\left(\theta_{j}-\theta_{a v}\right)^{2}+\text { h.o.t. }
\end{aligned}
$$

Comparing (57) and (59), we see that (56) holds for (any) $c_{2}>$ $(N+1) /(N)$ in the vicinity of the synchronised state and therefore also for some uniformly bounded constant $c_{2}$ in the compact set $\mathcal{N}$. Using the inequalities (55) and (56) in (54) yields

$$
\left.\dot{V}\right|_{\mathbf{v}=0} \leq-\kappa\left(1-N c_{1} c_{2}\|P t\|\right)\left\|\operatorname{grad} U_{1}\right\|^{2} .
$$

This implies that for every $\kappa>0$, there exists a neighborhood of the parallel equilibrium (46) where the Lyapunov function $V$ satisfies

$$
\left.\dot{V}\right|_{\mathbf{v}=0} \leq-\epsilon\left\|\operatorname{grad} U_{1}\right\|^{2}
$$

for some $\epsilon>0$. With $v_{k}=u_{k}-\left(\partial U_{1}\right) /\left(\partial \theta_{k}\right)$ defined from (50), one obtains

$$
\begin{aligned}
\dot{V} \leq & -\epsilon\left\|\operatorname{grad} U_{1}\right\|^{2} \\
& -\kappa \sum_{k=1}^{N}\left(\frac{\partial U_{1}}{\partial \theta_{k}}-\left\langle P_{k} \mathbf{t}, \frac{d_{k}}{d_{k}+1} i e^{i \theta_{k}}\right\rangle\right) v_{k} \\
= & -\epsilon\left\|\operatorname{grad} U_{1}\right\|^{2}-\sum_{k=1}^{N} v_{k}^{2} \leq 0 .
\end{aligned}
$$

For a fixed $\kappa>0$, let $\bar{V}_{\kappa}$ be the largest value such that (62) holds in the set $\Omega_{\kappa}=\left\{(\mathbf{t}, \boldsymbol{\theta}) \mid V(\mathbf{t}, \boldsymbol{\theta}) \leq \bar{V}_{\kappa}\right\}$. Then the set $\Omega_{\kappa}$ is invariant and solutions in $\Omega_{\kappa}$ converge to the largest invariant set where $\boldsymbol{\theta}=\theta_{0} \mathbf{1}$ for some $\theta_{0} \in S^{1}$ and $v_{k}=0, k=1, \ldots, N$. This is a set of parallel equilibria satisfying (51).

The phase control $\mathbf{u}=\operatorname{grad} U_{1}$ stabilizes the set of parallel equilibria, which is of dimension $2(N-1)$ in the shape space. Away from singularities, the $N$ algebraic constraints (51) are independent. As a result, the control law (50) isolates a subset of parallel equilibria of dimension $N-2$ in the shape space.

The result of Theorem 8 is thus weaker than the results for circular equilibria for two reasons: the result is only local and the control law does not isolate the desired parallel equilibrium for $N>2$. A simple calculation indeed shows that the Jacobian linearization of (52) at the parallel equilibrium (46) possesses $N-$ 2 uncontrollable spatial modes with zero eigenvalue. This means that the Jacobian linearization of the closed-loop system will possess $N-2$ zero eigenvalues for any smooth static state feedback. For $N>2$, no smooth static state feedback can achieve exponential stability of an isolated relative parallel equilibrium.

\section{Stabilizable Collectives}

In this section, we focus on the control structure (19) and discuss the role of key parameters. The constant $\omega_{0}$ determines the type of relative equilibrium. For $\omega_{0} \neq 0$, the control (19) produces circular motion with radius $\rho_{0}=\left|\omega_{0}\right|^{-1}$ and sense of rotation determined by the sign of $\omega_{0}$. The potential $U$ determines the steady-state phase arrangement. For $\omega_{0}=\kappa=0$ and $U=K U_{1}, K<0$, the control (19) produces parallel motion.

In the following subsections, we investigate removing the $\mathrm{SE}(2)$ symmetry of the control (19), i.e., its invariance to rigid translation and rotation in the plane [1]. We stabilize circular motion about a fixed beacon and parallel motion along a fixed reference direction. We define behavior primitives to enable the group to track piecewise-linear trajectories with fixed waypoints.

\section{A. $\mathrm{SE}(2)$ Symmetry Breaking}

The control (19) depends only on the relative spacing, $r_{k j}=$ $r_{k}-r_{j}$, and relative phase, $\theta_{k j}=\theta_{k}-\theta_{j}$, variables. As a result, the model (1) with control (19) is invariant to rigid translations 
and rotations in the plane, which corresponds to the action of the symmetry group $\mathrm{SE}(2)$. In this subsection, we investigate breaking this symmetry first by adding a fixed beacon to break the $\mathbb{R}^{2}$ symmetry and, second, by adding a heading reference to break the $S^{1}$ symmetry.

We break the $\mathbb{R}^{2}$ translation symmetry of the spacing control (19) by stabilizing circular motion with respect to a fixed beacon. Let $R_{0} \in \mathbb{C}$ be the location of a fixed beacon and (re)define the vector from the beacon to particle $k$ by $\tilde{r}_{k}=r_{k}-R_{0}$. We obtain the following extension of Theorem 3 .

Corollary 2: Consider the particle model (1) and a smooth phase potential $U(\boldsymbol{\theta})$ that satisfies $\langle\operatorname{grad} U, \mathbf{1}\rangle=0$. The control law (19) where $\tilde{r}_{k}=r_{k}-R_{0}, R_{0}$ is the location of a fixed beacon, and $U_{1}$ is removed, i.e.,

$$
u_{k}=\omega_{0}\left(1+\kappa\left\langle r_{k}-R_{0}, \dot{r}_{k}\right\rangle\right)-\frac{\partial U}{\partial \theta_{k}}, \quad \omega_{0} \neq 0
$$

enforces convergence of all solutions to a circular formation of radius $\rho_{0}=\left|\omega_{0}\right|^{-1}$ about $R_{0}$. Moreover, the asymptotic phase arrangement is a critical point of the potential $U$.

Proof: We use the Lyapunov function $V_{0}(\mathbf{r}, \boldsymbol{\theta})$ which is the sum of the composite Lyapunov function $V(\mathbf{r}, \boldsymbol{\theta})$ defined by (20) in the proof of Theorem 3 and a new term that is minimized when the center of the circular relative equilibrium is at $R_{0}$

$$
V_{0}(\mathbf{r}, \boldsymbol{\theta})=V(\mathbf{r}, \boldsymbol{\theta})+\frac{1}{2 N}\left\|\mathbf{1}^{T} \mathbf{s}+i N \omega_{0} R_{0}\right\|^{2} .
$$

Recall that

$$
V(\mathbf{r}, \boldsymbol{\theta})=\frac{\kappa}{2}\|P \mathbf{s}\|^{2}+U(\boldsymbol{\theta})
$$

with $\mathbf{s}$ given by (9) and $P$ the projector defined in (10). We compute

$$
\begin{aligned}
\dot{V}_{0}=\sum_{k=1}^{N}\left(\kappa \left\langleP_{k} \mathbf{s}+\right.\right. & \frac{1}{N} \mathbf{1}^{T} \mathbf{s} \\
& \left.\left.+i \omega_{0} R_{0}, i e^{i \theta_{k}}\right\rangle+\frac{\partial U}{\partial \theta_{k}}\right)\left(u_{k}-\omega_{0}\right) .
\end{aligned}
$$

Since

$$
P_{k} \mathbf{s}+\frac{1}{N} \mathbf{1}^{T} \mathbf{s}+i \omega_{0} R_{o}=e^{i \theta_{k}}-i \omega_{0}\left(r_{k}-R_{0}\right)
$$

using the control law (63), we once again obtain $\dot{V}_{0}=$ $-\sum_{k=1}^{N}\left(u_{k}-\omega_{0}\right)^{2} \leq 0$ along the solutions of the closed-loop system. Solutions converge to the largest invariant set $\Lambda$ where

$$
\kappa\left\langle\omega_{0} \tilde{r}_{k}, i e^{i \theta_{k}}\right\rangle=\frac{\partial U}{\partial \theta_{k}}
$$

for $k=1, \ldots, N$. Proceeding as in the proofs of Theorems 2 and 3, we use the result that on $\Lambda$ the control is $u_{k}=\omega_{0}$ for $k=1, \ldots, N$. This implies that the right hand side of (65) is constant for each $k$. Since $\dot{\theta}_{k}=\omega_{0}, \tilde{r}_{k} \neq 0$ and the left-hand side of (65) is constant only if it is zero. This implies a circular relative equilibrium centered at $R_{0}$.

Next, we break the $S^{1}$ rotational symmetry of the control (19) by introducing a heading reference $\theta_{0}$, where $\dot{\theta}_{0}=\omega_{0}$.
We couple the dynamics of the particle group to the reference heading by adding a new coupling term to only one of the particles in the group. This yields the following extension of Theorem 3.

Corollary 3: Consider the particle model (1) and a smooth phase potential $U(\boldsymbol{\theta})$ that satisfies $\langle\operatorname{grad} U, \mathbf{1}\rangle=0$. Let $u_{k}$, $k=1, \ldots, N-1$ be given by (19) and

$$
\begin{aligned}
& u_{N}=\omega_{0}\left(1+\kappa\left\langle\tilde{r}_{N}, \dot{r}_{N}\right\rangle\right)-\frac{\partial\left(U-\kappa U_{1}\right)}{\partial \theta_{N}} \\
& +d \sin \left(\theta_{0}-\theta_{N}\right)
\end{aligned}
$$

where $\dot{\theta}_{0}=\omega_{0}$ and $d>0$. This control enforces convergence of all solutions to relative equilibria as in Theorem 3. In addition, relative equilibria with phase arrangement minimizing $U$ and satisfying $\theta_{N}=\theta_{0}$ define an asymptotically stable set.

Proof: Consider the potential

$$
W(\mathbf{r}, \boldsymbol{\theta})=V(\mathbf{r}, \boldsymbol{\theta})+d\left(1-\cos \left(\theta_{0}-\theta_{N}\right)\right)
$$

with $V(\mathbf{r}, \boldsymbol{\theta})$ given by (20). The time-derivative of $W$ along the solutions of the particle model is

$$
\dot{W}=\dot{V}+d \sin \left(\theta_{0}-\theta_{N}\right)\left(\dot{\theta}_{0}-\dot{\theta}_{N}\right) .
$$

The control law (66) with $\dot{\theta}_{0}=\omega_{0}$ and $u_{k}$ given by (19) for $k=1, \ldots, N-1$ results in $\dot{W}=-\sum_{k=1}^{N}\left(u_{k}-\omega_{0}\right)^{2}$. Solutions therefore converge to the largest invariant set $\Lambda$ where (24) holds for $k=1, \ldots, N-1$. The $N-1$ equations $P_{k} \mathbf{s}=0, k=$ $1, \ldots, N-1$, imply $P \mathbf{s}=0$ because the matrix $P$ has rank $N-1$. Likewise, the $N-1$ equations $(\partial U) /\left(\partial \theta_{k}\right)=0, k=$ $1, \ldots, N-1$, imply $\operatorname{grad} U=0$ because the Hessian of $U$ has rank $N-1$. The relation $u_{N}=\omega_{0}$ then reduces to

$$
d \sin \left(\theta_{0}-\theta_{N}\right)=0
$$

which implies that $\theta_{N}=\theta_{0}$ or $\theta_{0}+\pi$. Therefore, relative equilibria with $\theta_{N}=\theta_{0}$ which minimize $V$ are asymptotically stable since they also minimize the potential $W$.

We note that in the case $\omega_{0}=0$, Corollary 3 proves asymptotic stability of parallel collective motion to a fixed heading reference, $\theta_{0}$. We use this result in the following subsection.

\section{B. Trajectory Tracking With Behavior Primitives}

We use the control (19) to define four behavior primitives which can be combined to track piecewise-linear trajectories following [18]. The behavior primitives include impulsive controls to align the particles with the reference input trajectory and feedback controls to stabilize this trajectory. The behaviors are referred to as circular-to-parallel, parallel-to-parallel, parallel-to-circular, and circular-to-circular. In parallel motion, the group center of mass follows a fixed reference heading. In the circular state, particles orbit a fixed beacon with a prescribed radius and sense of rotation.

Circular-to-Parallel: Starting from circular motion, this behavior stabilizes parallel motion along a fixed reference heading. The inputs to this behavior are the reference heading, 
$\theta_{0}$, and the gain, $d$. The impulse control which aligns the particles in the reference direction is

$$
\triangle \theta_{k}=\theta_{0}-\theta_{k} .
$$

The feedback control that stabilizes parallel motion is of the form (19) for $k=1, \ldots, N-1$ and (66) for $k=N$ with $\omega_{0}=\kappa=0$ and $U=K U_{1}, K<0$.

1) Parallel-to-Parallel: Starting from parallel motion, this behavior stabilizes parallel motion along a different reference trajectory. The inputs to this behavior are the new reference heading, $\theta_{0}$, and the gain, $d$. The impulsive control used to align the particles in the input direction is given by (70). The feedback control that stabilizes parallel motion is of the form (19) for $k=1, \ldots, N-1$ and (66) for $k=N$ with $\omega_{0}=\kappa=0$ and $U=K U_{1}, K<0$.

2) Parallel-to-Circular: Starting from parallel motion, this behavior stabilizes circular motion about the location of the center of mass at the time the behavior is initiated. The input to this behavior are the parameters, $\omega_{0} \neq 0$ and $\kappa>0$, the initial center of mass of the group, $R_{0}$, and the phase potential $U(\boldsymbol{\theta})$. The impulsive control used to align the particles in the input rotation direction is given by

$$
\triangle \theta_{k}=\arg \left(i \omega_{0} \tilde{r}_{k}\right)-\theta_{k}
$$

The feedback control used to stabilize circular motion is of the form (63), which is (19) with $\tilde{r}_{k}=r_{k}-R_{0}$, where $R_{0}$ is a fixed beacon and $U_{1}$ is removed.

3) Circular-to-Circular: Starting from circular motion, this behavior stabilizes circular motion with a different radius, i.e., dilation/contraction, about the same fixed reference. The input to this behavior are the parameters, $\omega_{0} \neq 0$ and $\kappa>0$, the initial center of mass of the group, $R_{0}$, and the phase potential $U(\boldsymbol{\theta})$. There is no impulsive control used to realign the particles. The feedback control used to stabilize circular motion is of the form (63), which is (19) with $\tilde{r}_{k}=r_{k}-R_{0}$, where $R_{0}$ is a fixed beacon and $U_{1}$ removed.

Next, we use the behavior primitives to construct a behavior sequence that tracks a sample reference trajectory. The admissible references are piecewise-linear paths specified by a list of desired heading and duration pairs. An example of trajectory tracking is shown in Fig. 5. In this example, twelve particles start from random initial conditions in the vicinity of the origin (point A). Each step in the behavior sequence is simulated for 200 time steps. The behavior sequence starts by stabilizing circular motion about the origin with $\omega_{0}=\kappa=1 / 25$, $R_{0}=0$, and $U(\boldsymbol{\theta})=0$. The next behavior in the sequence is circular-to-parallel with reference heading $\theta_{0}=\pi / 8$ and gain $d=1 / N$, which takes the sensor network from point A to point B in Fig. 5. At point B, the behavior parallel-to-parallel is used to track the reference input $\theta_{0}=-3 \pi / 8$ to point $\mathrm{C}$. Then the parallel-to-circular behavior stabilizes circular motion about a fixed center of mass with $\omega_{0}=-1 / 25, \kappa=\left|\omega_{0}\right|$, and $U(\boldsymbol{\theta})=0$. The sequence is repeated for the points $\mathrm{C}, \mathrm{D}$, and $\mathrm{E}$.

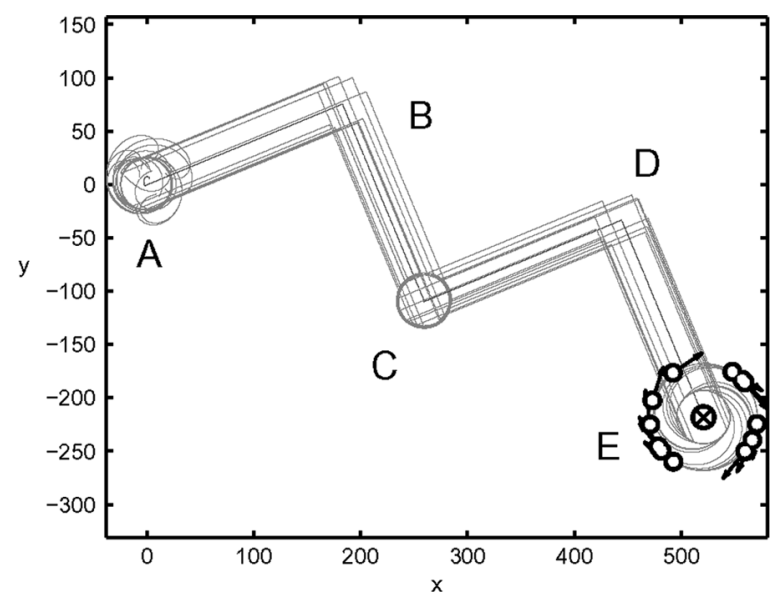

Fig. 5. Trajectory tracking with $N=12$ starting from random initial conditions. The reference input is a piecewise-linear curve. The behavior sequence starts in the vicinity of A by stabilizing circular motion with $\omega_{0}=1 / 25$ and then follows A circular-to-parallel, B parallel-to-parallel, and C parallel-tocircular. This sequence repeats for the points $\mathrm{C}, \mathrm{D}$, and $\mathrm{E}$ and then ends with the circular-to-circular behavior at $\mathrm{E}$ with $\omega_{0}=-1 / 50$. See text for control parameters.

Lastly, the circular-to-circular behavior stabilizes circular motion in the same direction with the new radius $\rho_{0}=\left|\omega_{0}\right|^{-1}=50$ and $U(\boldsymbol{\theta})=0$.

\section{Relevance and Limitations for Engineering Applications}

The proposed coordinated group trajectory design methodology has been developed on the basis of simplified models that may seem only remotely connected to the engineering applications presented in the introduction. We briefly discuss the relevance and the limitations of the proposed approach, in particular in the context of the specific ocean sampling application that motivated much of this work.

Models of point-mass particles steered at constant speed are of course a strong simplification of dynamic models. Our approach is to decouple the trajectory design problem, that we primarily view as a collective design problem, from the tracking control problem, that we primarily view as an individual design problem. This decoupling may not hold in full generality but seems at least reasonable in applications where the time-scale of the collective mission is significantly slower than the time-scale of the individual dynamics.

In such situations, we envision the use of our controlled models primarily in the task of trajectory design (and related collective optimization designs). This means that given an initial condition and a desired collective motion for the group, the simulated closed-loop trajectory of the model provides a reference trajectory for each of the vehicles. In such a scheme, each vehicle is equipped with an internal tracking controller whose task is to resolve the discrepancies between the reference and the actual trajectory. The natural place to use a more detailed model is in the design of the tracking controller of the individual vehicles. This hierarchical control scheme is common in applications. It does not necessarily imply that the design of the reference trajectories be offline and centralized. In fact, the whole purpose of the present work is to make the design of such reference trajectories adaptive and decentralized. This means 
that the initial conditions of the controller that produces the coordinated trajectory design will be continuously refreshed but typically at a slower time scale than the time scale of the individual tracking controllers. The all-to-all communication setting of the present paper limits application to a centralized path planning controller, but this limitation will be overcome in a companion paper.

In the ocean sampling application described in [10], we provide further details on the integration of the proposed design in a sensor network of underwater vehicles. In this application, the collective task is to maximize the information intake. Solving this dynamic optimization problem over the individual trajectories is a formidable and unrealistic task in a changing environment that involves several distinct time scales. We rather propose to restrict the optimization problem over the few parameters that define simple collective shapes like the ones proposed in this paper. We explicitly discuss an example involving several vehicles tracing elliptical shapes with prescribed relative spacing around the ellipses; this requires some generalization of the circular shapes discussed in the present paper. This setup has already been successfully demonstrated in a major field experiment with a fleet of ten autonomous underwater gliders in Monterey Bay, California, throughout the month of August 2006. We note that the all-to-all communication setting works best for this ocean sampling application because the vehicles do not communicate directly with one another but rather each communicates (albeit asynchronously) with a common central computer.

Decoupling the collective trajectory design problem from the individual reference tracking problem does not mean that the setup of the present paper is sufficient to address all of the many challenges of collective engineering applications. For instance, the issue of avoiding collisions (between vehicles or between vehicles and obstacles) is not addressed in the present paper. For the described ocean sampling application, the spatial scales are such that collision avoidance is not a primary issue for the trajectory generation controller. Rather, it is a design specification for the individual controllers. In other applications, such as collective flight in narrow formations, collision avoidance might be a primary issue that should be addressed at the level of the coordinated trajectory design. Control laws with this capability have been proposed in [1] and it is of interest in future research to include collision avoidance in the present setting.

There are several further stability and robustness issues that also deserve to be addressed at the group level and not only at the individual level. A concrete example in the context of the ocean sampling application is the issue of sea currents. In real ocean conditions, currents can be of the same magnitude or greater than the propulsion capability of some underwater vehicles. As a consequence, they should be taken into account even in the simplified models used for the trajectory design. Further collective measures, such as the string stability notion considered in the framework of vehicle platooning [29], may prove useful to assess the relevance of the proposed approach to engineering applications.

\section{CONCLUSION}

This paper proposes a design methodology to stabilize isolated relative equilibria in a model of all-to-all coupled identical particles moving in the plane at unit speed. The stabilizing feedbacks derive from potentials that reach their minimum in the desired configuration and possess no other identified local minima. Lyapunov analysis of the closed-loop system thus proves exponential stability of the desired equilibria and suggests almost global convergence properties.

Stabilization of the phase variables, $\theta_{k}$, is based on minimizing or maximizing successive momenta associated to the $N$ phasors, $e^{i \theta_{k}}$. The $m$-th moment is minimum when particle phases balance modulo $2 \pi / m$ and is maximum when phases synchronize modulo $2 \pi / m$. For parallel formations, maximizing the first moment results in synchronization of the orientations and a spacing potential can be added to (locally) correct the relative distances between particles. For circular formations, a spacing potential is proposed that reaches its minimum when all particles orbit the same point. This spacing potential is combined with the phase potentials in order to stabilize symmetric pattern circular configurations. The last section of the paper proposes a low-order parametrized family of stabilizable collectives that can be combined to solve path-planning or optimization problems at the group level.

The results of the paper rest on two idealistic assumptions: all-to-all communication and identical individuals. The assumption of all-to-all communication is completely relaxed in a companion paper [30] where we extend the present results to restricted communication topologies. The assumption of identical individuals is fundamental to the symmetry properties of the closed-loop vector field, but the exponential stability of isolated relative equilibria implies some robustness of the corresponding collective motions to individual variations. It is of interest to study in future work how the ideally engineered models considered in this paper may help in capturing gross dynamical properties of more realistic simulated multi-agent models or empirical observations of animal groups.

\section{REFERENCES}

[1] E. W. Justh and P. S. Krishnaprasad, "Equilibria and steering laws for planar formations," Syst. Control Lett., vol. 52, no. 1, pp. 25-38, 2004.

[2] J. A. Fax and R. M. Murray, "Information flow and cooperative control of vehicle formations," IEEE Trans. Autom. Control, vol. 49, no. 9, pp. 1465-1476, Sep. 2004.

[3] W. Ren and R. Beard, "Formation feedback control for multiple spacecraft via virtual structures," IEE Proc. Control Theory Appl., vol. 151, no. 3, pp. 357-368, 2004.

[4] L. Chaimowicz, V. Kumar, and F. Campos, "A paradigm for dynamic coordination of multiple robots," Auton. Robots, vol. 17, no. 1, pp. $7-21,2004$

[5] Y. Cao, A. Fukunaga, A. Kahng, and F. Meng, "Cooperative mobile robotics: Antecedents and directions," in Proc. IEEE/RSJ Int. Conf. Intell. Robots Syst., 1995, pp. 226-234.

[6] M. G. Earl and R. D'Andrea, "Multi-vehicle cooperative control using mixed integer linear programming," arXiv:cs/0501092v1 [CS.RO].

[7] J. Cortès, S. Martìnez, T. Karatas, and F. Bullo, "Coverage control for mobile sensing networks," IEEE Trans. Robot. Automat., vol. 20, no. 2, pp. 243-255, Apr. 2004.

[8] A. Bertozzi, M. Kemp, and D. Marthaler, "Determining environmental boundaries: Asynchronous communication and physical scales," in $\mathrm{Co}$ operative Control: 2003 Block Island Workshop on Cooperative Control, V. Kumar, N. Leonard, and A. S. Morse, Eds., 2005, pp. 25-42, New York: Springer-Verlag.

[9] E. Fiorelli, N. E. Leonard, P. Bhatta, D. Paley, R. Bachmayer, and D. M. Fratantoni, "Multi-AUV control and adaptive sampling in Monterey Bay," IEEE J. Ocean. Eng., vol. 31, no. 4, pp. 935-948, Oct. 2006. 
[10] N. E. Leonard, D. Paley, F. Lekien, R. Sepulchre, D. M. Fratantoni, and R. Davis, "Collective motion, sensor networks and ocean sampling," Proc. IEEE, vol. 95, no. 1, pp. 48-74, Jan. 2007.

[11] Y. Kuramoto, Chemical Oscillations, Waves, and Turbulence. New York: Springer-Verlag, 1984.

[12] A. Jadbabaie, J. Lin, and A. Morse, "Coordination of groups of mobile autonomous agents using nearest neighbor rules," IEEE Trans. Autom. Control, vol. 48, no. 6, pp. 988-1001, Jun. 2003.

[13] L. Moreau, "Stability of multiagent systems with time-dependent communication links," IEEE Trans. Autom. Control, vol. 50, no. 2, pp. 169-182, Feb. 2005.

[14] P. Newton, P. Holmes, and A. Weinstein, Geometry, Mechanics, and Dynamics. Volume in Honor of the 60th Birthday of J.E. Marsden. New York: Springer-Verlag, 2002.

[15] J. A. Marshall, M. E. Broucke, and B. A. Francis, "Formations of vehicles in cyclic pursuit," IEEE Trans. Autom. Control, vol. 49, no. 11, pp. 1963-1974, Nov. 1994.

[16] E. Justh and P. Krishnaprasad, A simple control law for UAV formation flying ISR, U. Maryland, Tech. Rep. 2002-38, 2002.

[17] R. Sepulchre, D. Paley, and N. E. Leonard, "Collective motion and oscillator synchronization," in Cooperative Control: 2003 Block Island Workshop on Cooperative Control, V. Kumar, N. Leonard, and A. S. Morse, Eds., 2005, pp. 189-228, New York: Springer-Verlag.

[18] D. Paley, N. E. Leonard, and R. Sepulchre, "Collective motion: Bistability and trajectory tracking," in Proc. 43rd IEEE Conf. Decision Control, 2004, pp. 1932-1937.

[19] S. H. Strogatz, "From Kuramoto to Crawford: Exploring the onset of synchronization in populations of coupled oscillators," Physica D, vol. 143 , pp. $1-20,2000$.

[20] H. Levine, W.-J. Rappel, and I. Cohen, "Self-organization in systems of self-propelled particles," Phys. Rev. E, vol. 63, p. 017101, 2000

[21] B. Ermentrout and N. Kopell, "Oscillator death in systems of coupled neural oscillators," SIAM J. Appl. Math., vol. 50, pp. 125-146, 1990.

[22] F. Hoppensteadt and E. Izhikevich, Weakly Connected Neural Networks. New York: Springer-Verlag, 1997.

[23] S. Watanabe and S. Strogatz, "Constants of motion for superconductor arrays," Physica D, vol. 74, pp. 197-253, 1994.

[24] E. Brown, J. Moehlis, and P. Holmes, "On phase reduction and response dynamics of neural oscillator populations," Neural Comput., vol. 16, no. 4, pp. 673-715, 2004.

[25] I. Couzin, J. Krause, R. James, G. Ruxton, and N. Franks, "Collective memory and spatial sorting in animal groups," J. Theor. Biol., vol. 218, pp. 1-11, 2002.

[26] T. Vicsek, A. Czirók, E. Ben-Jacob, I. Cohen, O. Shochet, and A. Tenenbaum, "Novel type of phase transition in a system of self-driven particles," Phys. Rev. Lett., vol. 75, pp. 1226-1229, 1995.

[27] D. Paley, N. E. Leonard, and R. Sepulchre, "Oscillator models and collective motion: Splay state stabilization of self-propelled particles," in Proc. 44th IEEE Conf. Decision Control, 2005, pp. 3935-3940.

[28] K. Okuda, "Variety and generality of clustering in globally coupled oscillators," Physica D, vol. 63, pp. 424-436, 1993.

[29] D. Swaroop and J. K. Hedrick, "String stability of interconnected systems," IEEE Trans. Autom. Control, vol. 41, no. 3, pp. 249-256, Mar. 1996.

[30] R. Sepulchre, D. Paley, and N. E. Leonard, "Stabilization of planar collective motion: limited communication,"IEEE Trans. Autom. Control, to be published.

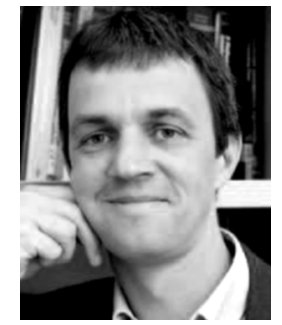

Rodolphe Sepulchre (M'96) received the engineering and Ph.D. degrees in applied mathematics from the University of Louvain, Belgium, in 1990 and 1994, respectively.

$\mathrm{He}$ is a Professor in the Department Electrical Engineering and Computer Science, Université de Liège, Institut Montefiore, Liège, Belgium. From 1994 to 1996, he held a postdoctoral position at the University of California, Santa Barbara. In 2002-2003, he held a visiting position at Princeton University, Princeton, NJ. His research focuses on theory and applications of nonlinear dynamical systems. He is coauthor with M. Jankovic and P. Kokotovic of the book Constructive Nonlinear Control (New York: Springer-Verlag, 1997). He serves as an Associate Editor for the journals Mathematics of Control, Signals and Systems, and SIAM Journal of Control and Optimization.

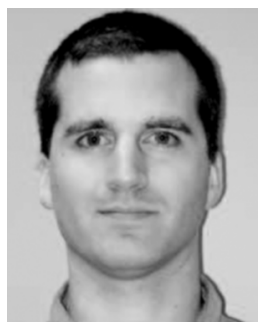

Derek A. Paley (S'02) received the B.S. degree in applied physics from Yale University, New Haven, CT, in 1997, and the M.A. degree in mechanical and aerospace engineering from Princeton University, Princeton, NJ, in 2004. He is a currently working toward the Ph.D. degree in the Department of Mechanical and Aerospace Engineering, Princeton University.

From 1997 to 2000, he worked as an analyst in the defense industry for Metron, Inc., Reston, VA. From 2000 to 2002, he worked as a Software Engineer in the underwater vehicle industry for Bluefin Robotics Corp., Cambridge, MA. His research interests are collective motion of natural and engineered systems, cooperative control, and ocean sampling with underwater vehicles.

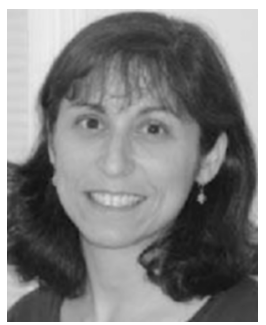

Naomi Ehrich Leonard (S'90-M'95-SM'02-F'07) received the B.S.E. degree in mechanical engineering from Princeton University, Princeton, NJ, in 1985, and the M.S. and Ph.D. degrees in electrical engineering from the University of Maryland, College Park, in 1991 and 1994, respectively.

She is a Professor of Mechanical and Aerospace Engineering and an Associated Faculty member of the Program in Applied and Computational Mathematics at Princeton University. From 1985 to 1989, she worked as an Engineer in the electric power industry for MPR Associates, Inc. Her current research interests include nonlinear control and dynamics, cooperative control, mobile sensor networks, autonomous underwater vehicles, adaptive ocean sampling, and collective motion in animal aggregations. 